\title{
Risk factors related to the loss of reduction after acromioclavicular joint dislocation treated with the EndoButton device
}

\author{
Kai Chen, Bin Xu, Yang-Jun Lao, Yang Yang \\ Department of Orthopaedics, Tongde Hospital of Zhejiang, Hangzhou, China \\ Contributions: (I) Conception and design: K Chen; (II) Administrative support: Y Yang; (III) Provision of study materials or patients: K Chen; (IV) \\ Collection and assembly of data: YJ Lao; (V) Data analysis and interpretation: B Xu; (VI) Manuscript writing: All authors; (VII) Final approval of \\ manuscript: All authors. \\ Correspondence to: Yang Yang, MM. Department of Orthopaedics, Tongde Hospital of Zhejiang Province, no. 234 Gu-cui Road, Hangzhou 310012 , \\ China. Email: 251859502@qq.com.
}

\begin{abstract}
Background: Anatomic ligament reconstruction using the EndobutBon device has been proven an effective technique for the management of acute acromioclavicular (AC) joint dislocation. The aim of this study was to analyze the risk factors causing loss of reduction after AC joint dislocation treated with the EndoButton device for open procedures.

Methods: A total of 107 patients who met the inclusion and excluded criteria finally were enrolled. We retrospectively analyzed the clinical tests, imaging data, and postoperative rehabilitation of patients in a stable reduction group and a loss of reduction group.

Results: Among all patients included in the study, the incidence of reduction failure was $14 \%$. Univariate analysis identified that the time between injury to surgery, double or triple EndoButton, coracoid EndoButton position, weight-bearing time of the upper limb, and osteolysis had statistically significant associations with loss of reduction for the $\mathrm{AC}$ joint $(\mathrm{P}=0.022,0.002,0.000,0.000$, and 0.000 respectively). By using multivariate logistic regression analysis, we found that coracoid EndoButton position $(\mathrm{P}=0.002)$, weight-bearing time of the upper $\operatorname{limb}(\mathrm{P}=0.011)$, and osteolysis $(\mathrm{P}=0.036)$ were the significant independent risk factors related to the loss of reduction after AC joint dislocation treated with the EndoButton device used with open procedures.

Conclusions: In order to reduce the incidence of reduction failure and improve the prognosis of patients, more accurate placement of EndoButton plates in surgery and guidance for patients in completing planned rehabilitation training are needed.
\end{abstract}

Keywords: Acromioclavicular joint dislocation (AC joint dislocation); EndoButton technique; loss of reduction; risk factor

Submitted Nov 27, 2020. Accepted for publication Feb 10, 2021.

doi: $10.21037 /$ atm-21-404

View this article at: http://dx.doi.org/10.21037/atm-21-404

\section{Introduction}

There are more than dozens of surgical methods for the treatment of acute acromioclavicular (AC) joint dislocation; however none can be regarded as the current gold standard. These different schemes do share a concept based on anatomical coracoclavicular (CC) ligament reconstruction technology $(1,2)$. These anatomical techniques typically involve cortical fixation devices (3-5), free tendon grafts (6), or a combination of both (7), each of which require holes to be drilled into the distal clavicle and/or the coracoid process. The EndoButton technique is among the methods that biomechanical studies have consistently shown to provide sufficient biomechanical strength for patients with AC joint dislocation, with most patients achieving satisfactory prognosis $(8,9)$. However, with the continuous 
extension of follow-up time, a series of complications such as loss of reduction, osteolysis, wire cutting of the steel plate, iatrogenic fracture of the clavicle or coracoid process, heterotopic ossifications, and others have been reported in quick succession (4,8,10-12). Improper surgical indications, unsuitable surgical methods, erroneous selection of the EndoButton, inaccurate positioning, and other some minor operational errors in the operation can cause corresponding complications, with some patients even requiring a second revision operation (13). Thus far, no studies have analyzed and compared all the proposed risk factors for complications of the Endobutton device.

We conducted a systematic retrospective study on patients with AC joint dislocation, summarized and recorded all the possible risk factors, and conducted statistical analysis, to ultimately identify the statistically significant risk factors. It is hoped that if these risk factors can be avoided in clinical work, the incidence of complications can be reduced, with patients benefitting from a better prognosis.

We present the following article in accordance with the STROBE reporting checklist (available at http://dx.doi. org/10.21037/atm-21-404).

\section{Methods}

\section{Patients}

We retrospectively analyzed the age, sex, BMI index, time between injury and operation, and Rockwood classification of patients who underwent EndoButton device surgery in our hospital from February 2014 to February 2017. Other risk variables were also recorded, including the use of double or triple EndoButton technique, alignment of the EndoButton, position of the coracoid EndoButton (central, medial, or lateral), weight-bearing time of the upper $\operatorname{limb}(<6$ or $>6$ weeks), heterotopic ossifications, and osteolysis. We used strict inclusion and exclusion criteria for all patients. The inclusion criteria were as follows: (I) age range 18-65 years, (II) Rockwood type III, IV, and V dislocations, (III) complete follow-up data, (IV) EndoButton device with open surgery technique. The exclusion criteria were the following: (I) combined with basic diseases, (II) complicated with multiple injuries, (III) vascular and nerve injury on the injured side.

All procedures performed in this study involving human participants were in accordance with the Declaration of Helsinki (as revised in 2013). The study was approved by the Ethics Committee of Tongde Hospital of Zhejiang and informed consent was taken from all the patients.

\section{Procedure}

All patients underwent surgery that included the double EndoButton (2) or triple EndoButton technique (4) as performed by the same group of surgeons in our department (a chief physician and an attending physician with more than 2 years of experience). Arthroscopically assisted techniques were not included in the procedure.

In the Endobutton procedure, the injured shoulder was immobilized in a brace at $30^{\circ}$ of abduction and at neutral position for 6 weeks. Analgesia was performed by cold compress and oral or intravenous drug on the first day after surgery, with non-steroidal anti-inflammatory drugs (NSAIDs) having been administered for the previous 6 weeks after operation. After their pain had subsided, the patients could begin shoulder joint pendulum exercises. Passive training was mainly used for the first 4 postoperative weeks, with the range of abduction and flexion not exceeding $90^{\circ}$. Active training was generally performed after postoperative week 4 , gradually reaching the full range of shoulder joint activities. All patients were told to avoid lifting weight for up to 6 weeks postoperatively.

\section{Data}

The imaging data we collected and evaluated included radiologic examinations (anterior-posterior, axillary and bilateral shoulder contrast of Zanca views) at the time of injury, radiology reviews the day after surgery, and radiologic examinations from multiple follow-up visits in the outpatient department. We used a picture archiving and communication system (PACS) to record our measurements. The main outcome variable was loss of reduction, which was defined as an upward clavicle displacement exceeding $6 \mathrm{~mm}$ (7). The position of the coracoid EndoButton device was defined as central, medial, or lateral according to the anterior and posterior shoulder X-ray scans after the operation (see Figure 1). Heterotopic ossifications and osteolysis were identified according to the postoperative or final follow-up anterior-posterior views of the injured shoulder.

\section{Statistical analysis}

The measurement data in this study are expressed as mean and standard deviation, and were analyzed using one- 


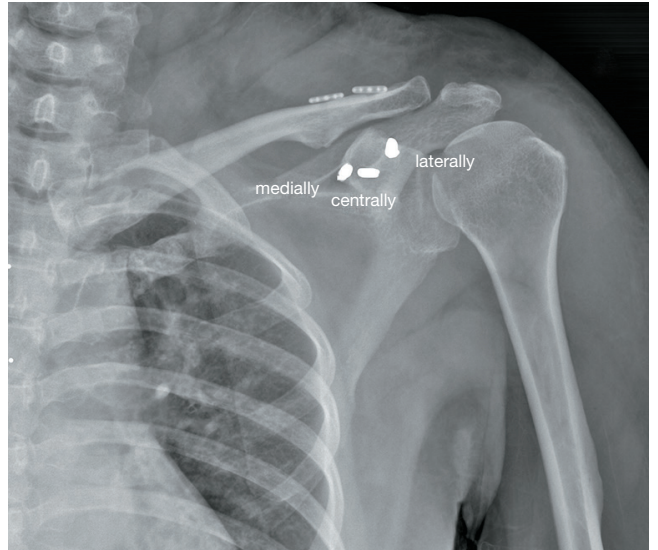

Figure 1 The position of coracoid endobutton plate (central, medial, lateral).

way ANOVA. Counting data are expressed as percentage and were analyzing using a chi-square test. The examined variables in a loss of reduction group were compared with those of a stable reduction group. After the variables with statistical significance were selected, multivariate logistic regression analysis was performed to exclude the related interference factors. Finally, the factors that had significant significance for the loss of reduction were selected, and the relationship between these factors was identified and tested. All analyses were performed using SPSS software, v.23.0 (IBM Corp., Armonk, NY, USA).

\section{Results}

A total of 107 patients, including 60 males and 47 females, were included in this study. The average follow-up time was 24.3 months and the median age was 46.70 years. There were 35 Rockwood type III cases, 42 type IV cases, and 30 type V cases. The mean time from injury to operation was 4.85 days (range, 1-12 days). The average BMI was $22.94 \mathrm{~kg} / \mathrm{m}^{2}$.

For the main outcome variable, 15 of 107 (14.0\%) patients had loss of reduction. In the loss of reduction group, 3 patients were managed with the triple EndoButton technique, and the other 12 patients were treated with the double EndoButton technique; 10 of the 15 patients had the coracoid button placed laterally and 3 had it placed medially (Figure 2 and Figure 3) to the center of the coracoid base; 7 of 15 patients (46.7\%) were identified to have osteolysis; 12 of the 15 patients began upper limb weight-bearing within 6 weeks postoperatively, while the other 3 patients began after 6 weeks; heterotopic ossifications were detected in 2
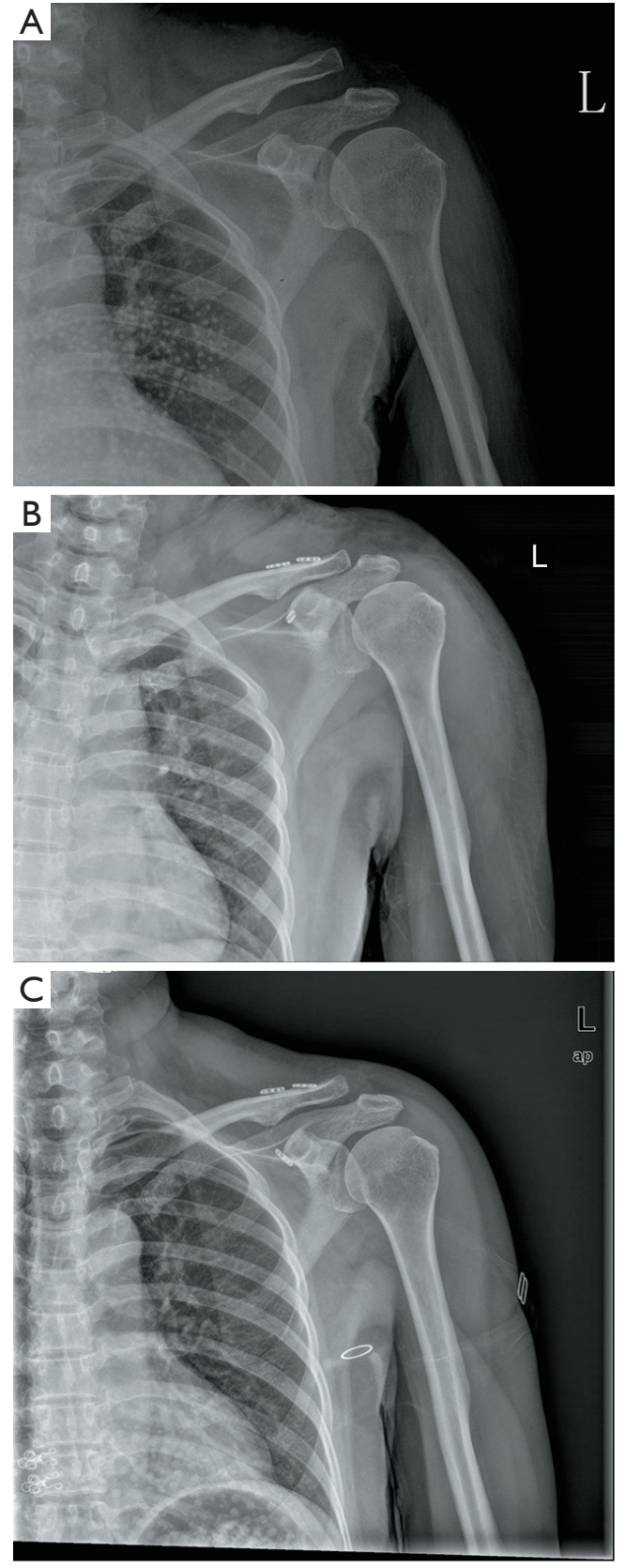

Figure 2 loss of reduction because the medial position of the coracoid button. (A) A 50-year-old woman with AC joint dislocation (Rockwood type III). (B) X-ray examination on the second day after operation showed that the reduction was good, but the coracoid button was placed too far medially. (C) Two months after operation, the reduction was lost. 


\section{Page 4 of 8}
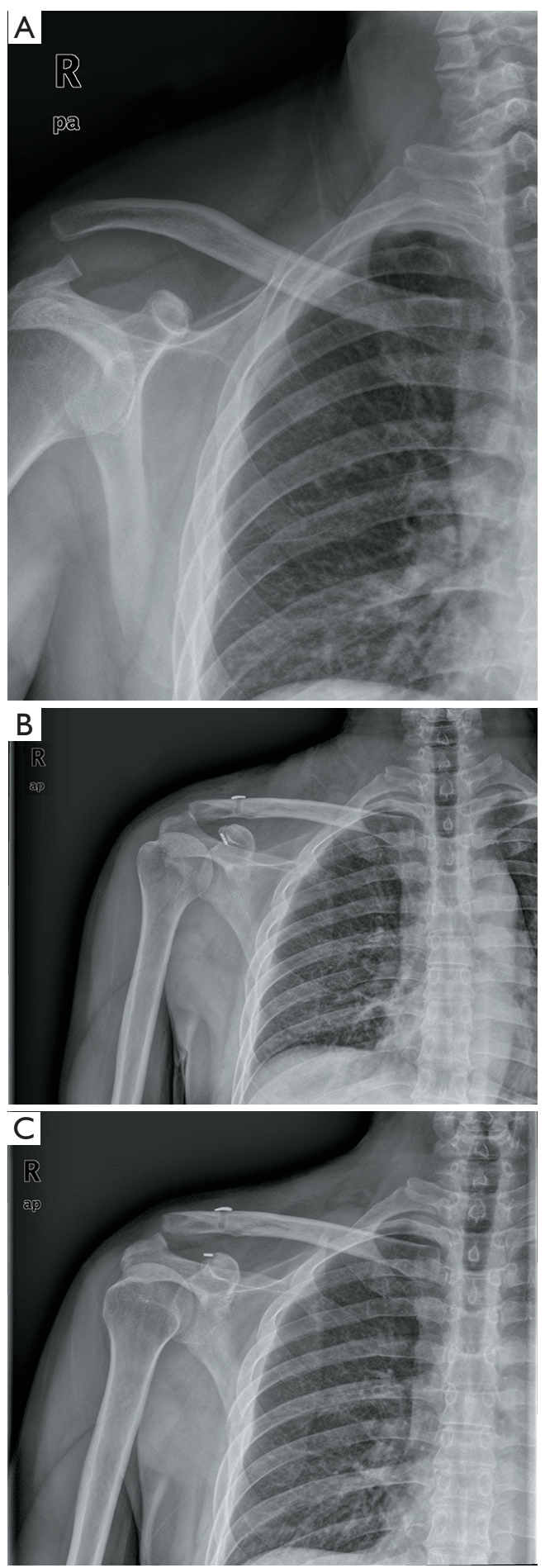

Figure 3 loss of reduction because the lateral position of the coracoid button. (A) A 38-year-old man with AC joint dislocation (Rockwood type III). (B) Double EndoButton technique was performed. Two days after the operation. X-ray film showed a good reduction of the AC joint, but the coracoid button was placed too far laterally. (C) A loss of reduction was found 1 month after surgery.
Chen et al. Risk factors for loss of reduction after AC joint dislocation

cases $(1.9 \%)$.

Univariate analysis showed that the time between injury to surgery, double or triple EndoButton technique, coracoid EndoButton position, weight-bearing time of the upper limb, and osteolysis were significantly different between the two groups $(\mathrm{P}=0.022,0.002,0.000,0.000$, and 0.000 respectively) (Table 1).

The results of multivariate logistic regression analysis are shown in Table 2. The results showed that there were significant differences in the position of the coracoid button $(\mathrm{P}=0.002)$, osteolysis $(\mathrm{P}=0.036)$, and weight-bearing time of the upper $\operatorname{limb}(\mathrm{P}=0.011)$ between the two groups.

\section{Discussion}

To our knowledge, this is the first study to systematically analyze and compare all the proposed risk factors related to the loss of reduction after AC joint dislocation managed with the EndoButton device using open procedures. Although some authors have speculated on and proposed several factors associated with loss of reduction for AC joint dislocation, single factor analysis of this kind often leads to erroneous conclusions due to the interference of various factors. Therefore, our study used multivariate logistic regression analysis to exclude the interference from factors and obtain a more accurate conclusion. The most important finding of the present study was that the significant risk factors for loss of reduction were the position of the coracoid button, the weight-bearing time of the upper limb, and osteolysis.

Both medial and lateral dislocations of the coracoid button may increase the probability of postoperative loss of reduction. In the loss of reduction group, 10 of the 15 patients had the coracoid button placed laterally and 3 had it placed medially to the center of the coracoid base. This is in line with the conclusions of other authors $(7,11,14,15)$. One factor that might contribute to this is the deviation of positioning and drilling position due to improper steps taken during the operation. Research by Ferreira et al. (14) has shown that center-to-center coracoid tunnel drilling is an ideal surgical technique that offers a reduced risk of postoperative reduction loss. We believe that during the operation, the first step should be to drill a bone tunnel in the central part at the base of the coracoid process, followed by excessive reduction to the AC joint, and drilling a hole into the clavicle; this can achieve a relatively accurate positioning and avoid the deviation of direction as much as possible. This misplacement of the coracoid button caused 
Table 1 A comparison of possible risk factors between the loss of reduction and stable reduction groups

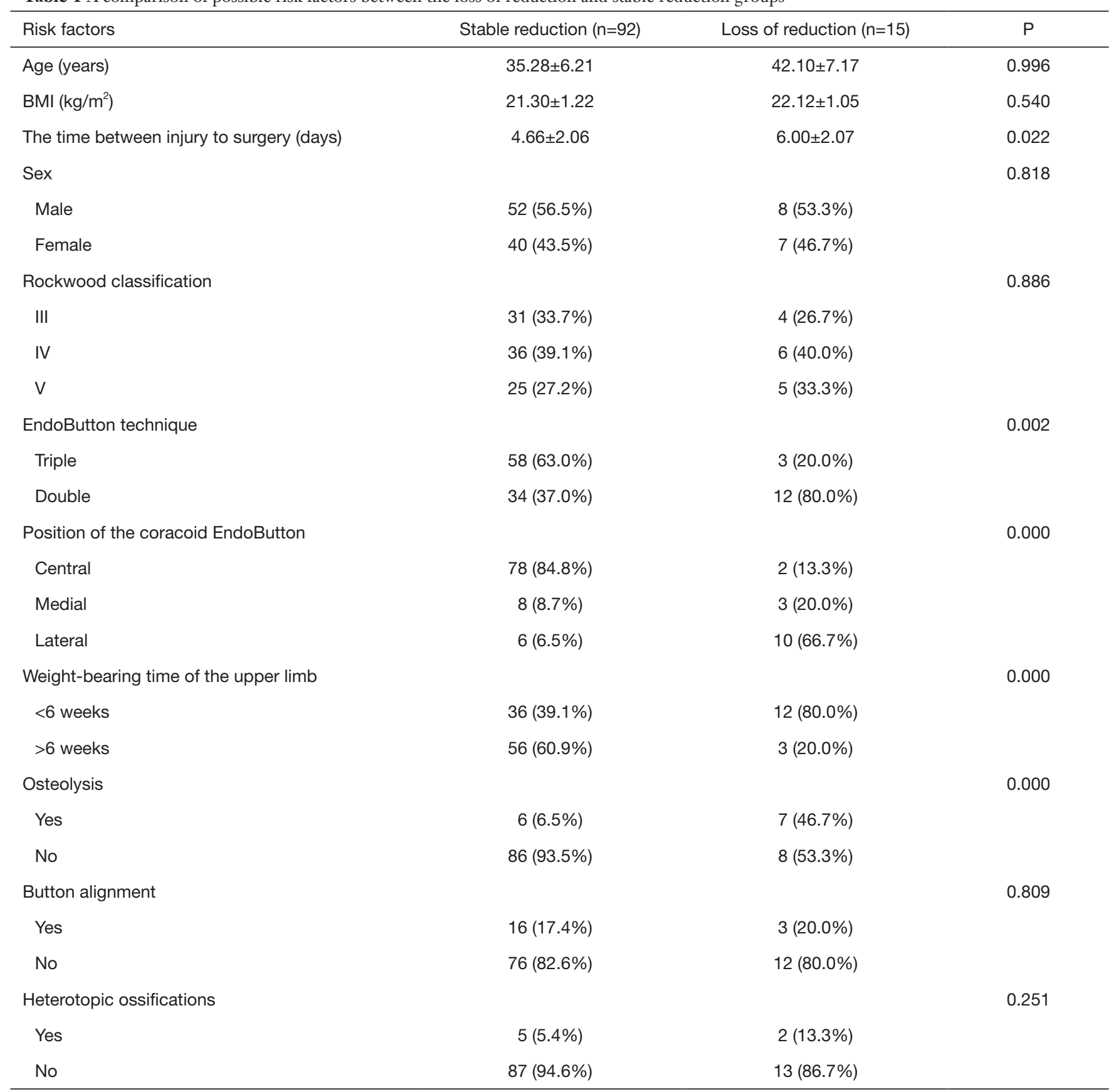

by the operative procedure might eventually lead to an increased loss of reduction (11). Undeniably, the surgeons experience is an important factor in determining whether or not the drilling of the coracoid tunnel is in the correct position or not. When drilling, we prefer using a bit with a smaller diameter, as a bit that is too thick can damage the bone of the coracoid process and clavicle, leading to a loss of bone mass. In addition, we find plate alignment to not be a significant risk factor affecting reduction loss, which is not the view held by other researchers (11), for whom the position of the button seems to be more important.

Osteolysis was another risk factor associated with loss of reduction. Although few studies have analyzed the development of clavicle osteolysis as a risk factor for loss of reduction, it was 
Table 2 Multivariate logistic regression analysis

\begin{tabular}{|c|c|c|c|c|c|c|}
\hline Risk factors & B & S.E. & Wald & $d f$ & Sig. & $\operatorname{Exp}(B)$ \\
\hline EndoButton technique & 1.514 & 1.280 & 1.400 & 1 & 0.237 & 4.545 \\
\hline Position of the coracoid EndoButton & -2.536 & 0.818 & 9.614 & 1 & 0.002 & 0.079 \\
\hline Weight-bearing time of the upper limb & -3.162 & 1.250 & 6.400 & 1 & 0.011 & 0.042 \\
\hline
\end{tabular}

found to be an independent factor in our study. Osteolysis of the distal clavicle can occur for several reasons, including the direct violent injury of the AC joint and repeated excessive weight-bearing on the upper limb (16). In addition, as reported by many studies, regardless of internal fixation use, AC joint dislocation itself may cause osteolysis (17-19). From our statistical analysis, 13 of 107 patients were identified as having distal clavicle osteolysis, 7 of whom had a loss of reduction. The appearance of osteolysis ranged from 2 weeks to 10 months after the operation in our study. Osteolysis may appear within 1 month of the initial injury, when the ligament healing has not been completed (15). The clavicular and acromial branches of the thoracoacromial artery are located in the lateral four-fifth of the clavicle and provide blood supply to the soft tissue and bone, so any injury to this vessel during the surgical procedure may cause clavicle osteolysis (20). Consequently, care should be taken during the surgical procedure to avoid damaging the clavicular and acromial vessel, especially the periosteal vascularity and branches of the thoracoacromial artery.

In the present study, the risk of loss of reduction was significantly increased when the time upper limb weightbearing began earlier than 6 weeks after surgery. In the loss of reduction group, 12 of the 15 patients began upper limb loading before the postoperative week 6 , while the other 3 patients began after 6 weeks. A portion of patients did not follow the doctor's medical orders and began upper limb loading before 6 weeks had passed. These patients generally had occupations involving heavy physical labor. Before the 6-week period had elapsed, the ligament might not have completely healed by the time the patients began upper limb loading, and frequent stress stimulation might also have contributed to this. Clayton et al. (21) concluded that the sutured ligament had "functionally normal strength" after 6 weeks, while Frank et al. (22) reported an experiment about characteristics of ligament that demonstrated significantly increased laxity at 3 weeks after injury, decreasing only slightly at 6 weeks. Studies (1) have shown that the soft tissue around the AC joint and the shackle gap is scarred 4-6 weeks after surgery, and this may confer a protective effect on the sutured tissue and the plate. According to the current multivariate analysis, we believe undertaking upper limb weight-bearing too early (less than 6 weeks) may induce the loss of reduction, and recommend that patients strictly avoid subjecting the injured upper limb to weight-bearing for at least 6 weeks after the operation.

Our analysis found that age, sex, BMI, time between injury and surgery, Rockwood classification, EndoButton technique, button alignment, and heterotopic ossifications had no significant correlation with the loss of reduction. Similarly, other authors have suggested that Rockwood classification, time between trauma and surgery, and heterotopic ossifications had no impact on the loss of reduction (11). Struhl et al. (8) also reported that button selection, time of surgery, soft tissue procedure, and distal clavicle intervention did not significantly affect radiographic outcomes. These results were basically consistent with our conclusion.

Some limitations to our study should be addressed. All the patients included in our analysis were from the same hospital, and thus our conclusions may not be applicable to other populations. Furthermore, the sample size of our study was generally low, and thus our results should be interpreted cautiously. Thirdly, all the operations in our study were performed using an open approach, and arthroscopically assisted techniques were not included in the procedure. Further investigation is necessary to determine whether an arthroscopically assisted EndoButton technique might reduce the failure rate.

\section{Conclusions}

Using retrospective study and statistical analysis, we found that the significant risk factors for postoperative loss of reduction after AC joint dislocation treated with the EndoButton device using open procedures were the 
position of the coracoid button, early upper limb weightbearing ( $<6$ weeks), and osteolysis. Accurate placement of the EndoButton plates and adherence to a rehabilitation plan are important factors in preventing loss of reduction.

\section{Acknowledgments}

Funding: None.

\section{Footnote}

Reporting Checklist: The authors have completed the STROBE reporting checklist. Available at http://dx.doi. org/10.21037/atm-21-404

Data Sharing Statement: Available at http://dx.doi. org/10.21037/atm-21-404

Conflicts of Interest: All authors have completed the ICMJE uniform disclosure form (available at http://dx.doi. org/10.21037/atm-21-404). The authors have no conflicts of interest to declare.

Ethical Statement: The authors are accountable for all aspects of the work in ensuring that questions related to the accuracy or integrity of any part of the work are appropriately investigated and resolved. All procedures performed in this study involving human participants were in accordance with the Declaration of Helsinki (as revised in 2013). The study was approved by the Ethics Committee of Tongde Hospital of Zhejiang and informed consent was taken from all the patients.

Open Access Statement: This is an Open Access article distributed in accordance with the Creative Commons Attribution-NonCommercial-NoDerivs 4.0 International License (CC BY-NC-ND 4.0), which permits the noncommercial replication and distribution of the article with the strict proviso that no changes or edits are made and the original work is properly cited (including links to both the formal publication through the relevant DOI and the license). See: https://creativecommons.org/licenses/by-nc-nd/4.0/.

\section{References}

1. Grutter PW, Petersen SA. Anatomical acromioclavicular ligament reconstruction: a biomechanical comparison of reconstructive techniques of the acromioclavicular joint. Am J Sports Med 2005;33:1723-8.
2. Costic RS, Labriola JE, Rodosky MW, et al. Biomechanical Rationale for Development of Anatomical Reconstructions of Coracoclavicular Ligaments After Complete Acromioclavicular Joint Dislocations. Am J Sports Med 2004:32:1929-36.

3. Struhl S. Double EndoButton Technique for Repair of Complete Acromioclavicular Joint Dislocations. Tech Shoulder Elbow Surg 2007;8:175-9.

4. Wei HF, Chen YF, Zeng BF, et al. Triple endobuttton technique for the treatment of acute complete acromioclavicular joint dislocations: preliminary results. Int. Orthop 2011;35:555-9.

5. Venjakob AJ, Salzmann GM, Gabel F, et al. Arthroscopically Assisted 2-Bundle Anatomic Reduction of Acute Acromioclavicular Joint Separations: 58-Month Findings. Am J Sports Med 2013;41:615-21.

6. Milewski MD, Tompkins M, Giugale JM, et al. Complications related to anatomic reconstruction of the coracoclavicular ligaments. Am J Sports Med 2012;40:1628-34.

7. Cook JB, Shaha JS, Rowles DJ, et al. Clavicular Bone Tunnel Malposition Leads to Early Failures in Coracoclavicular Ligament Reconstructions. Am J Sports Med 2013;41:142-8.

8. Struhl S, Wolfson TS. Continuous Loop Double EndoButton Reconstruction for Acromioclavicular Joint Dislocation. Am J Sports Med 2015;43:2437-44.

9. Grantham C, Heckmann N, Wang L, et al. A biomechanical assessment of a novel double EndoButton technique versus a coracoid cerclage sling for acromioclavicular and coracoclavicular injuries. Knee Surg Sports Traumatol Arthrosc 2016;24:1918-24.

10. Li Q, Hsueh PL, Chen YF. Coracoclavicular Ligament Reconstruction: a systematic review and a biomechanical study of a triple EndoButton technique. Medicine 2014;93:e193.

11. Schliemann B, Roßlenbroich SB, Schneider KN, et al. Why does minimally invasive coracoclavicular ligament reconstruction using a flip button repair technique fail? An analysis of risk factors and complications. Knee Surg Sports Traumatol Arthrosc 2015;23:1419-25.

12. Martetschläger F, Horan MP, Warth RJ, et al. Complications after anatomic fixation and reconstruction of the coracoclavicular ligaments. Am J Sports Med 2013;41:2896-903.

13. Tian J, Yu H, Xue H, et al. Failure cause of treating acromioclavicular joint dislocation by EndoButton technique. Chin J Bone Joint Surg 2012;06:48-51. 
14. Ferreira JV, Chowaniec D, Obopilwe E, et al. Biomechanical Evaluation of Effect of Coracoid Tunnel Placement on Load to Failure of Fixation During Repair of Acromioclavicular Joint Dislocations. Arthroscopy 2012;28:1230-6.

15. Sun LJ, Lu D, Chen H. Triple-Endobutton technique for the treatment of Tossy type III acromioclavicular joint dislocation. Zhongguo Gu Shang 2015;28:496-9.

16. Cahill BR. Osteolysis of the distal part of the clavicle in male athletes. J Bone Joint Surg Am 1982;64:1053-8.

17. Eschler A, Gradl G, Gierer P, et al. Hook plate fixation for acromioclavicular joint separations restores coracoclavicular distance more accurately than PDS augmentation, however presents with a high rate of acromial osteolysis. Arch Orthop Trauma Surg 2012;132:33-9.

18. Ye G, Peng CA, Sun HB, et al. Treatment of Rockwood type III acromioclavicular joint dislocation using

Cite this article as: Chen $\mathrm{K}, \mathrm{Xu} \mathrm{B}$, Lao YJ, Yang Y. Risk factors related to the loss of reduction after acromioclavicular joint dislocation treated with the EndoButton device. Ann Transl Med 2021;9(4):345. doi: 10.21037/atm-21-404 autogenous semitendinosus tendon graft and EndoButton technique. Ther Clin Risk Manag 2016;12:47-51.

19. Alentorn-Geli E, Santana F, Mingo F, et al. Distal Clavicle Osteolysis after Modified Weaver-Dunn's Procedure for Chronic Acromioclavicular Dislocation: A Case Report and Review of Complications. Case Rep Orthop 2014;2014:953578.

20. Lemos MJ, Tolo ET. Complications of the treatment of the acromioclavicular and sternoclavicular joint injuries, including instability. Clin Sports Med 2003;22:371-85.

21. Clayton ML, Weir GJ. Experimental investigations of ligamentous healing. Am J Surg 1959;98:373-8.

22. Frank C, Woo LY, Amiel D, et al. Medial collateral ligament healing. A Multidisciplinary assessment in rabbits. Am J Sports Med 1983;11:379-89.

(English Language Editor: J. Gray) 